\title{
Management of dentin dysplasia and facial disharmony
}

D ENTIN DYSPLASIA was first reported in 1922 by Ballschmiede ${ }^{1}$ who described six children in one family whose teeth had short, blunted roots with pulpal occlusion that he called "rootless teeth." Rushton ${ }^{2}$ later described a similar condition in an individual without evidence of genetic inheritance that he labeled "dental dysplasia." Cases have been reported in the literature that describe dentin dysplasia in association with a variety of congenital syndromes including familial calcinosis, ${ }^{3}$ Ehlers-Danlos syndrome, ${ }^{4}$ and brachio-skeleto-genital syndrome. ${ }^{5}$

In 1973, Shields and others ${ }^{6}$ described two types of dentin dysplasia. They defined type 1 as predominantly affecting the radicular portion of the teeth and generally characterized by foreshortened roots, pulpal occlusion, and periapical radiolucent areas. Type 2 was reported to be distinguished by opalescent primary teeth, followed by secondary teeth with flame-shaped pulp chambers containing denticles. Morris and Augsburger ${ }^{7}$ later described dentin dysplasia (type 1) associated with sclerotic bone and skeletal anomalies, and inherited as an autosomal dominant trait in a brother and sister. Eastman and others ${ }^{8}$ also described several patients with a focal dentinal dysplasia (type 3) in which only isolated teeth were affected. Although both light and scanning electron microscopy have demonstrated characteristic morphologic patterns for dentin dysplasia, ${ }^{9}$ Melnick and others ${ }^{10}$ state that the "pathogenesis ... continues to be an enigma."

From a review of the literature, it seems that dentin dysplasia may appear with or without predictable genetic heritability and that it may vary in clinical expression, both in isolation and with other congenital anomalies.

What follows is a report of a case involving combined dentin dysplasia, partial anodontia, and maxillary hypoplasia problems managed by the hospital dentistry team.

\section{REPORT OF CASE}

A 15-year-old white female without history of physical problems and appearing to have generally normal growth and development had been receiving routine dental care since early childhood. Her chief problem related to the appearance of her teeth. Following the dentist's suggestion, she consulted several specialists. She had recently been wearing maxillary and mandibular "bite-raising"' appliances presumably to determine her ability to tolerate a greater vertical dimension. She was subsequently referred by a local oral surgeon to the hospital dentistry team for consultation.

\section{Dental examination}

Intraoral examination showed a mixture of primary and secondary teeth with limited caries experience that had been controlled by regular dental care. The gingival crevice depth was less than $3 \mathrm{~mm}$ throughout, and signs of gingival inflammation were absent. At contact, vertical dimension and maxillary permanent first molars contacted the mandibular second molars while the teeth anterior to the molars remained out of contact (Fig 1). The following teeth were present: maxillary right first and

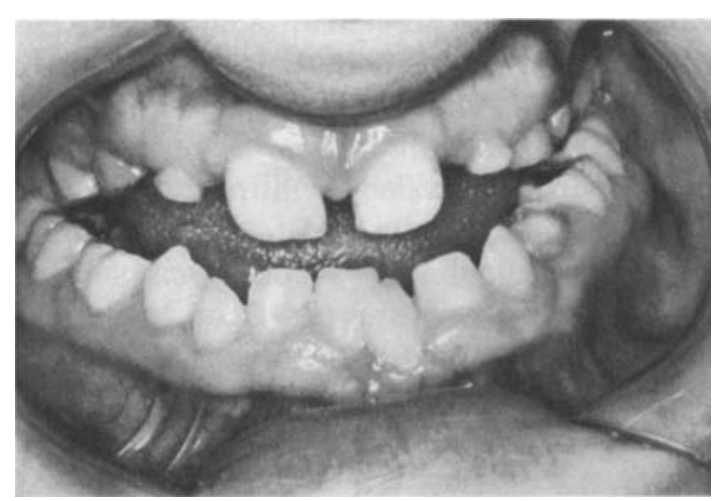

Fig 1. Mixed dentition with Class III molar relationship and anterior open bite.

\section{HOSPITAL}

DENTISTRY

\section{Patients with unusual congenital dental anomalies are often referred to the hospital dentistry team for evaluation and treatment.}

Special Care in Dentistry

Vol. 3 No. 3 May-June 1983 

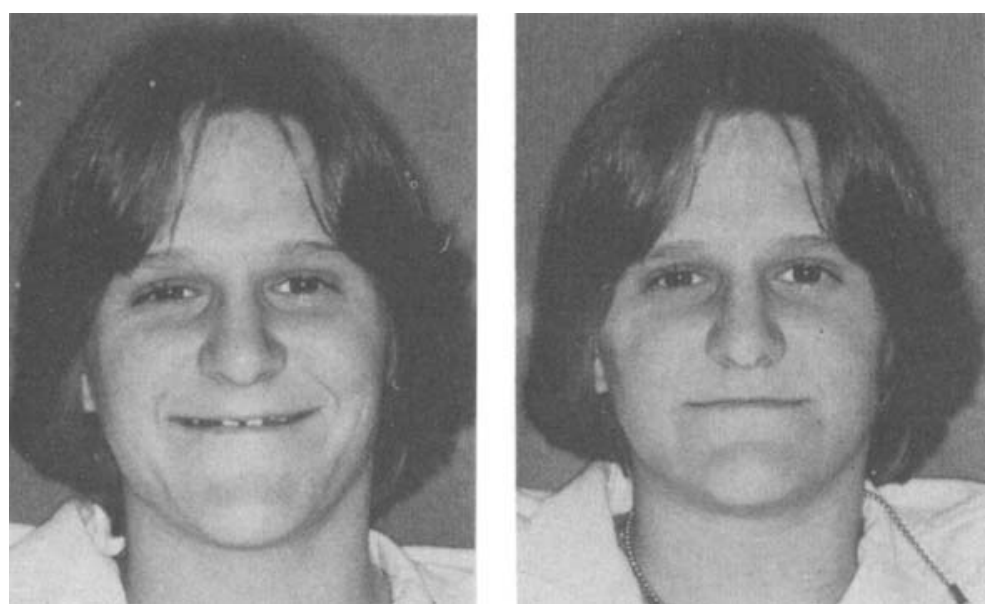

Fig 2. Pre-reconstruction facial view at rest (right); pre-reconstruction facial view at contact vertical dimension (left).

HOSPITAL DENTISTRY

Dentin dysplasia may appear with or without predictable genetic heritability and may vary in clinical expression

Special Care in Dentistry

Vol. 3 No. 3 May-June 1983 second molars, right primary molars, right primary lateral incisor, permanent central incisors, left primary lateral incisor, left primary canine, left primary first molar, left permanent second premolar, and left permanent first molar; mandibular right permanent first and second molars, right primary second molar, right primary and permanent canine, permanent incisors, left primary canine, left primary first and second molars, left permanent first and second molars.

The Class III facies of this patient was further enhanced by overclosure and a prominent chin. However, the primary skeletal anomaly was maxillary hypoplasia (Fig 2).

\section{Radiographic findings}

Periapical radiographs showed that, although some of the teeth displayed the classic foreshortened roots and occluded pulps, others possessed only diminutive root forms with pulps intact (Fig 3). The fully erupted permanent molar teeth, although "taurodont-like," appeared to have a more normal crown to root ratio and pulp chambers (Fig 4). None of the teeth showed the periapical radiolucent areas described by Shields and others, although occlusal contacts were not present on any of the affected teeth. Tooth structures representing the permanent maxillary right premolar, right and left canine, left premolar and second molar, and mandibular left second premolar and third molars were unerupted or impacted. There was no evidence intraorally or radiographically for the following permanent teeth: maxillary lateral incisors, maxillary third molars, mandibular left canine, and mandibular right premolars.

Lateral cephalogram showed a maxillary hy- poplasia with an associated vertical dimension loss that appeared to have been induced by reduction of dentoalveolar structure associated with the partial anodontia. The deficiency in dentoalveolar structure in the mandible was not reflected in the basal bone development (Fig 5).

\section{Treatment plan}

Orthodontic therapy was immediately eliminated from the choices of treatment because of the undeveloped root structure.

Orthognathic surgery for the maxilla to increase vertical facial height with bone grafting was considered. However, because of the developmental status of the remaining dentoalveolar structures, the effect of surgery on any further maxillary growth, the potential for disturbance to the few remaining teeth, and the ultimate dependence on a dental prosthesis, the surgeon suggested that formidable surgical intervention was not justified. Reduction genioplasty to reduce the chin prominence was suggested as a useful adjunct to prosthetic reconstruction of facial balance.

Prosthodontic alternatives were also limited because only the permanent molars were candidates for fixed restorations. Because of the patient's age and the many years of potential bone resorption, the favorable periodontal status of the mandibular teeth, and the difficulty in manipulating a mandibular removable appliance, the prosthodontist selected a maxillary overdenture as the final treatment.

\section{Dental therapy}

Initially, replacement denture teeth were set to a baseplate to determine if an acceptable esthetic result could be obtained without extrac-

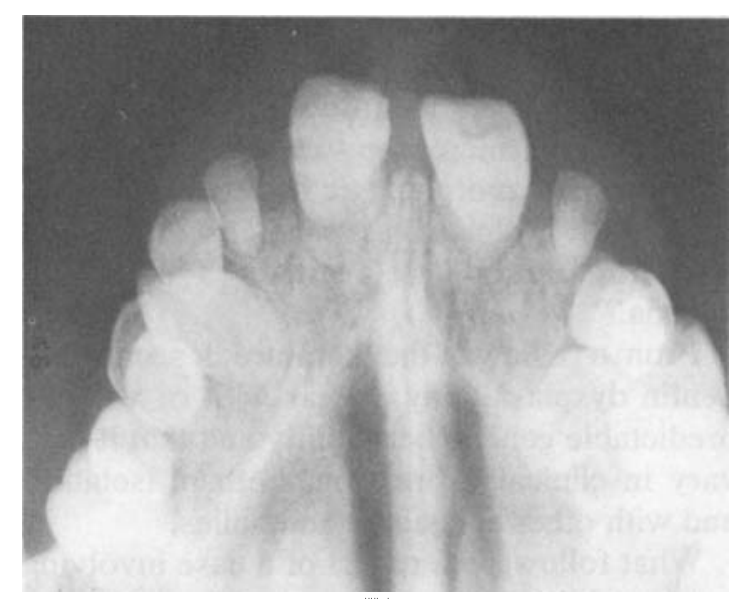

Fig 3. Primary and permanent incisors show foreshortened roots with pulpal occlusion. 


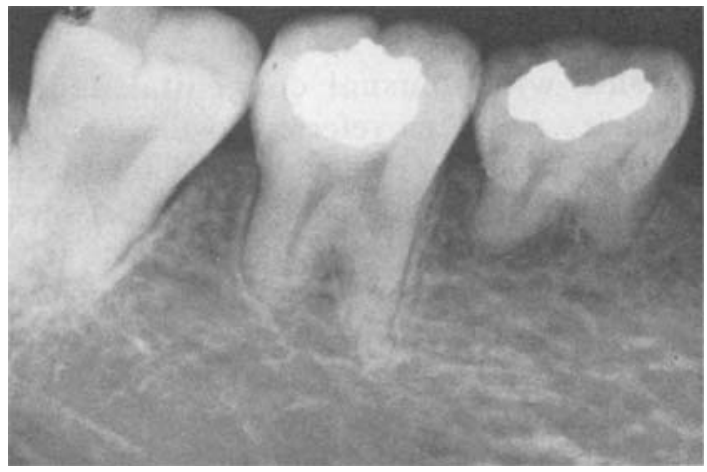

Fig 4. "Taurodont-like" permanent molars show more normal crown to root ratios.

tion of maxillary permanent teeth. When it was determined that there was adequate room for the replacement teeth, the baseplate was returned to the diagnostic mounting on which it was fabricated, and wax-up was completed with a posterior bite plane to open the vertical dimension and increase occlusal posterior contacts. The resulting trial denture was processed

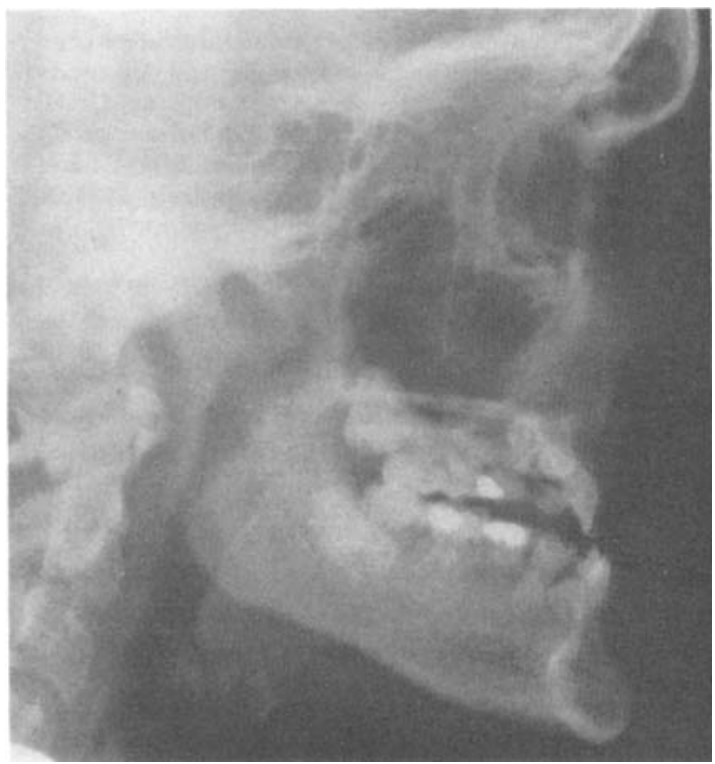

Fig 5. Lateral cephalogram shows deficient maxilla and prominent pogonion.

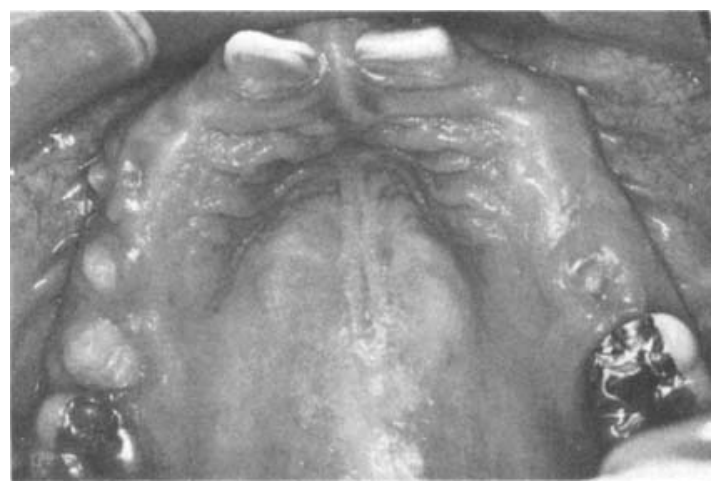

Fig 6. Maxillary molar crowns to restore optimal occlusal vertical dimension and retention for prosthesis. in heat-cured acrylic resin. To encourage eruption of underlying permanent tooth structures and because of the lack of root structures, all maxillary teeth-maxillary right primary molars, primary lateral incisors, left primary canine, and primary first molar-were extracted. The processed trial denture, to be worn until final prosthesis was completed, was lined with temporary soft reline material during surgery.

The first step in definitive reconstruction was full crown restoration of the maxillary permanent first molars (Fig 6). The occlusal surface of these teeth was increased to restore an acceptable vertical dimension and buccal contours were developed for clasping the final overdenture. Contouring the enamel of the maxillary central incisors allowed for a reasonable line of draw for the removable prosthesis because of the rather severe labial soft tissue undercuts. A cast metal framework with full palatal coverage, meshwork covering the edentulous ridge areas, and bilateral molar circumferential clasps, provided a stable definitive prosthesis easily modified to accommodate for eruption of the underlying permanent teeth (Fig 7).

The patient found the esthetic appearance of the final prosthetic appliance acceptable and chose to defer the reduction genioplasty (Fig $8)$.

\section{DISCUSSION}

Although this patient had attained physical maturity and was ready for definitive dental treatment, physically and psychologically her long-term dental needs were also important in selecting a final treatment plan. The initial tendency to overtreat this patient was avoided. Although supported by minimal root structure, removal of permanent teeth in good health simply to accommodate removable prostheses and maximize occlusal interdigitation could not be advised, especially because chewing was not a problem. Perhaps with good oral hygiene, the mandibular teeth affected by the congenital malformation will remain periodontally sound for many years. If not, the better developed molar teeth may be used to support a removable partial denture. In the interim, the unerupted teeth may continue to develop and the presently periodontally sound mandibular teeth will allow for conservation of bone. Surgical solutions may be more appropriate at a later time when jaw growth and development of dental structures are complete.

\section{HOSPITAL} DENTISTRY

The patient's

physical and psychological long-term dental needs were important in selecting a treatment plan.
Special Care in Dentistry

Vol. 3 No. 3

May-June 1983 

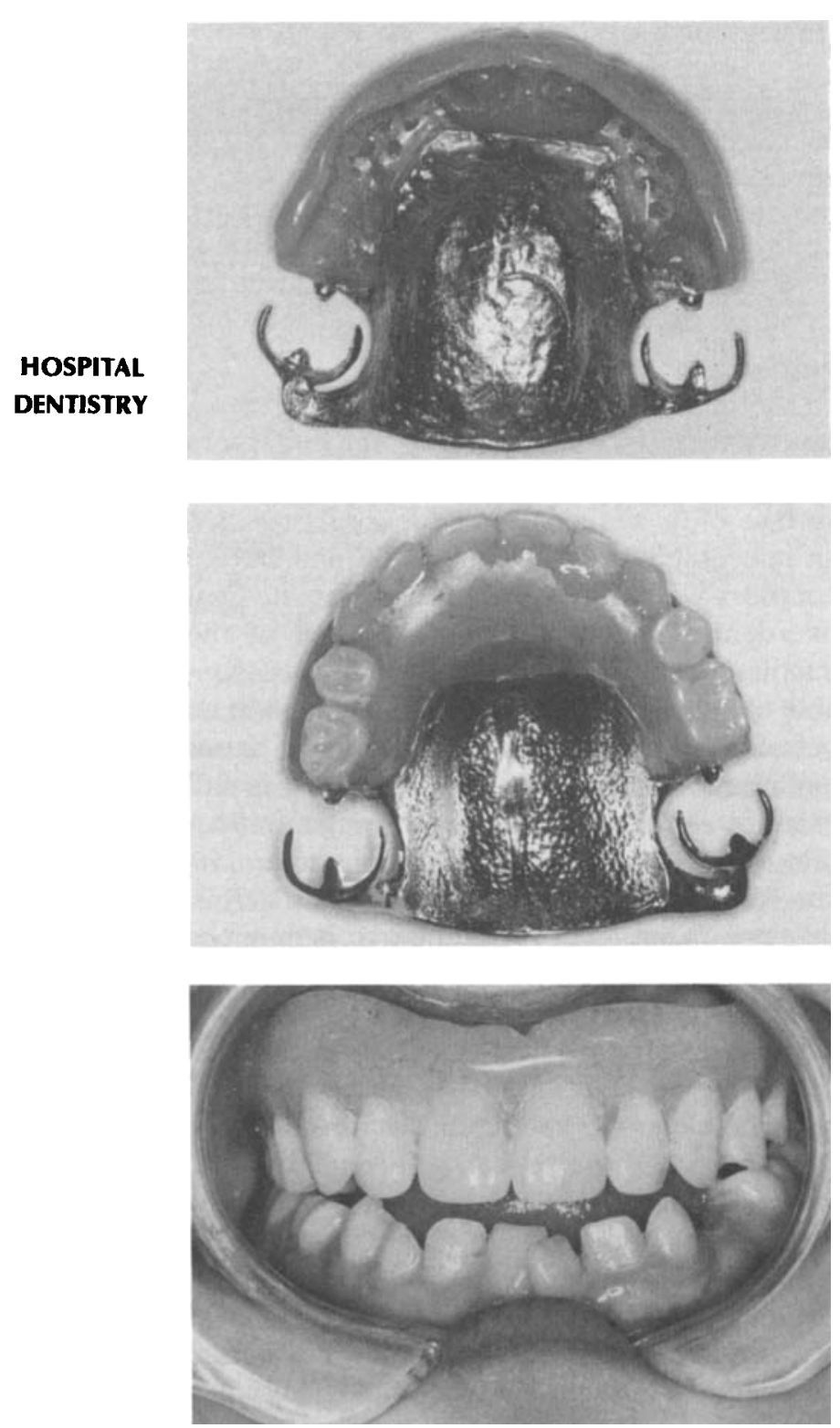

Fig 7. Final prosthesis.
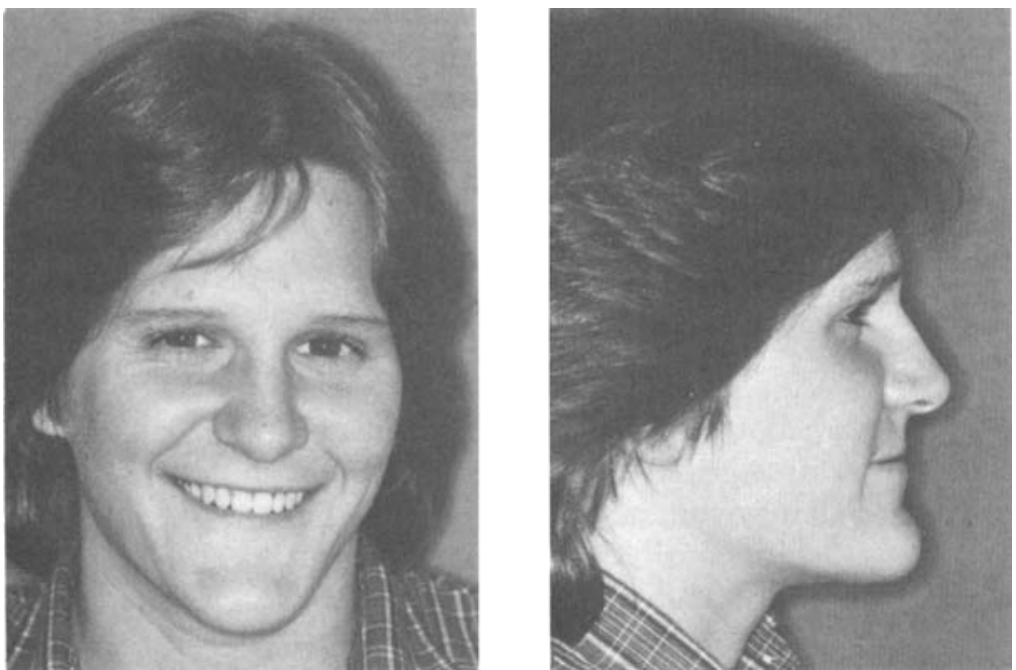

Fig 8. Post-reconstruction facial view at rest (left); post-reconstruction profile at contact vertical dimension (right).

\section{SUMMARY}

Patients with unusual congenital dental anomalies are often referred to the hospital dentistry team for evaluation and treatment recommendations. Communication between oral surgeon, orthodontist, pedodontist, periodontist, and prosthodontist after a combined single examination may simplify the treatment alternatives and save the patient the frustration and confusion of seeking treatment from a number of specialists. When necessary, combined treatment can be efficiently coordinated at the original consultation appointment. The recommendations of each specialist are then recorded together in the hospital record. This simplifies follow-up treatment and allows for easy transfer of information should the patient move to another location. In complex treatment cases, the pooling of dental specialist resources may permit conservatism and answer the patient's needs with lifetime perspectives.

Dr. Chamberlain is a maxillofacial prosthodontist at the University of Michigan Hospitals and assistant professor at the University of Michigan School of Dentistry, Ann Arbor, 48109; and Dr. Hayward is an oral surgeon recently retired from his practice at the University of Michigan Hospitals and as chairman, department of oral and maxillofacial surgery, University of Michigan School of Dentistry, Ann Arbor. Address requests for reprints to Dr. Chamberlain.

1. Ballschmiede, G. Beitragzurkasuistik der zahnanomalien in bezug auf zahl und grosse. Inang Diss Griefswald, 1922 H 50, 1922 , p 751 .

2. Rushton, M.A. A case of dentinal dysplasia. Guys Hosp Rep 89:369-373, 1939.

3. Hossins, G.S., and Marsland, E.A. Developmental abnormalities of the dentine and pulp associated with calcinosis. Br Dent J 92(12):305-311, 1952.

4. Barabas, G.M. The Ehlers-Danlos syndrome. Abnormalities of the enamel, dentine, cementum and the dental pulp: an histological examination of 13 teeth from 6 patients. Br Dent J 126(11):509$515,1969$.

5. Elsahy, N.I., and Waters, R.W. The brachio-skeleto-genital syndrome: a new hereditary syndrome. Plast Reconstr Surg 48(6):542-550, 1971.

6. Shields, E.D.; Bixler, D.; and el-Kafrawy, A.M. A proposed classification for heritable human dentine defects with a description of a new entity. Arch Oral Biol 18(4):543-553, 1973.

7. Morris, M.E., and Augsburger, R.H. Dentine dysplasia with sclerotic bone and skeletal anomalies inherited as an autosomal dominant trait: a new syndrome. Oral Surg 43(2):267-283, 1977.

8. Eastman, J.R.; Melnick, M.; and Goldblatt, L.I. Focal odontoblastic dysplasia: dentin dysplasia type III? Oral Surg 44(6):909914, 1977.

9. Witkop, C.J., Jr. Hereditary defects of dentin. In Poole, A.E., ed. Genetics of North America. Philadelphia, W. B. Saunders Co, 1975, pp 25-45.

10. Melnick, M.; Levin, L.S.; and Brady, J. Dentin dysplasia: a type 1: a scanning electron microscopic analysis of the primary dentition. Oral Surg 50(4):335-340, 1980. 